Military Technical College, Kobry El-Kobbah, Cairo, Egypt

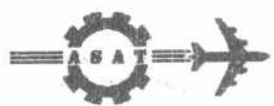

$9^{\text {th }}$ International Conference

On Aerospace Sciences \&

Aviation Technology

\title{
TEST OF THE PERFORMANCE OF AN OPTICAL SYSTEM
}

A. O. ELFarouk*, I. A. Ali", S. M. S. Saad"

\section{ABSTRACT}

The total MTF is a very powerful tool and comprehensive test of the performance of the optical system. In guidance and control of the missiles, it will express the ability of the homing head (seeker) to distinguish between the targets, different clutters and backgrounds specially, if there is a camouflaged background. The problem, which may face the investigator, would be the huge number of calculations to achieve an acceptable accuracy level.

This paper is directed towards building up a computer-based program capable of computing and plotting the Modulation Transfer Function (MTF).

\section{KEY WORDS}

The Modulation Transfer Function (MTF), The Optical Transfer Function (OTF), and The Phase Transfer Function (PTF), Infrared (IR).

\section{INTRODUCTION}

A type of target commonly used to test the performance of an optical system consists of a series of alternating light and dark bars of equal width, as indicated in Fig. (1a). Several sets of patterns of different spacing are usually imaged by the system under test and the finest set in which the line structure can be discerned is considered to be the limit of resolution of the system, which is expressed as a certain number of lines per millimeter. When a pattern of this sort is imaged by an optical system, each geometric line (i.e., of infinitesimal width) in the object is imaged as a blurred line, whose cross section is the line spread function. Fig. (1b) indicates a cross section of the brightness of the bar object, and Fig. (1c) shows how the image spread function "rounds off" the "corners" of the image.

* Egyptian Armed Forces 
In Fig. (1d), the effect of the image blur on progressively finer patterns is indicated. It is apparent that when the illumination contrast in the image is less than the smallest amount that the system (e.g., the eye, film, or photo detector) can detect, the pattern can no longer be "resolved". [1, 2]

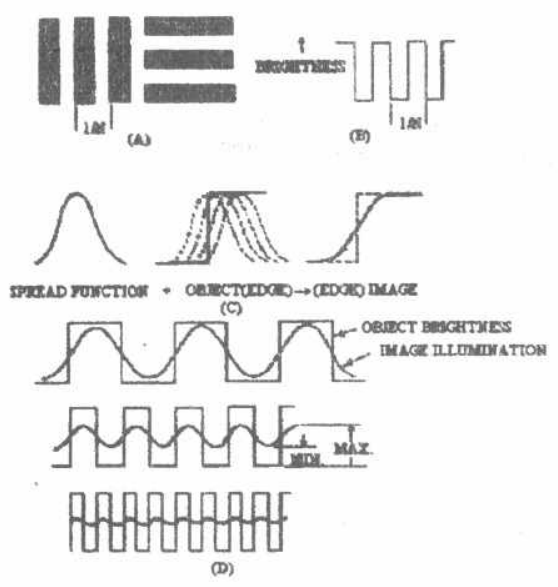

Fig. 1 the imagery of bar targets.

\section{COMPUTATION OF THE MODULATION TRANSFER FUNCTION (MTF)}

The preceding discussion has been based on patterns whose brightness distribution is a "square wave" Fig. (1b) and whose image illumination distribution is distorted or "rounded off" by characteristics of the optical system, as indicated in Fig. (1b). However, if the object pattern brightness distribution is in the form of a sine wave, the distribution in the image is also described by a sine wave, regardless of the shape of the spread function.

This fact has led to the widespread use of the modulation transfer function (MTF) to describe the performance of a lens system. The modulation transfer function is the ratio of the modulation in the image to that in the object as a function of the frequency (cycles per unit of length) of the sine-wave pattern.

Where:

$$
\operatorname{MTF}(v)=M / M \text { 。 }
$$

$M_{\mathrm{i}}$ : is the modulation in the image.

$M_{0}$ : is the modulation in the object. 
A plot of MTF against frequency $v$ is thus an almost universally applicable measure of the performance of an image-forming system and can be applied not only to lenses but to films, phosphors, image tubes, the eye, and even to complete systems such as camera-carrying aircraft. $[2,3,4]$

One particular advantage of the MTF is that it can be cascaded by simply multiplying the MTFs of two or more components to obtain the MTF of the combination. For example, if a camera lens with an MTF of 0.5 at 20 cycles per millimeter is used with a film with an MTF of 0.7 at this frequency, the combination will have an MTF of $0.5 \times 0.7=0.35$. If the object to be photographed with this camera has a contrast (modulation) of 0.1 , then the image modulation is $0.1 \times 0.35=0.035$, close to the limit of visual detection.

It should be noted, however, that MTFs do not cascade between optical components, which are directly "connected," i.e., lenses which are not separated by diffuser of some sort. This is because the aberrations of one component may compensate for the aberrations in another, and thus produce an image quality for the combination, which is superior to that of either component. Any "corrected" optical system illustrates this point.

The MTF has been referred to as frequency response, sine wave response, or contrast transfer.

Assume an object consisting of alternating light and dark bands, the brightness (luminance, radiance of which varies according to a cosine (or sine) function, as indicated by the upper part of Fig. (2), the distribution of brightness $(G(x))$ can be expressed mathematically by:

$$
\mathrm{G}(\mathrm{x})=\mathrm{b}_{0}+\mathrm{b}_{1} \cos (2 \pi v x)
$$

Where

$v \quad$ : is the frequency of the brightness variation in cycles per unit length,

$\left(b_{0}+b_{1}\right)$ : is the maximum brightness,

$\left(b_{0}-b_{1}\right)$ : is the minimum brightness, and

$x \quad$ : is the spatial coordinate perpendicular to the bands.

The modulation of this pattern is then given by:

$$
M_{0}=\frac{(b 0+b 1)-(b 0-b l)}{(b 0+b 1)+(b 0-b 1)}=\frac{b l}{b 0}
$$

When this line pattern is imaged by an optical system, each point in the object will be imaged as a blur. The energy distribution within this blur will depend on the relative aperture of the system and the aberrations present. Since we are dealing with a linear object, the image of each line element can be described by the line spread function as $A(\delta)$. We now assume (for convenience) that the dimensions $x$ and $(1 / v)$ in Eq. (1) are the corresponding dimensions in the image. It is apparent that the image energy distribution at a position $x$ is the summation of the product of $G(x)$ and $A(\delta)$ and can be expressed as follows

$$
\mathrm{F}(\mathrm{x})=\int \mathrm{A}(\delta) \mathrm{G}(\mathrm{x}-\delta) \mathrm{d} \delta
$$




\section{Where}

$F(x)$ is the image energy distribution at position $\mathrm{x}$.

Combining Eqs. (1) and (3), we get

$$
\mathrm{F}(\mathrm{x})=\mathrm{b}_{0} \int \mathrm{A}(\delta) \mathrm{d} \delta+\mathrm{b}_{1} \int \mathrm{A}(\delta) \cos [2 \pi \pi(\mathrm{x}-\delta)] \mathrm{d} \delta
$$

After normalizing by dividing by $\int A(\delta) d \delta$, Eq. (4) can be transformed to

$$
\begin{aligned}
\mathrm{F}(\mathrm{x}) & =\mathrm{b}_{0}+\mathrm{b}_{1}|\mathrm{~A}(v)| \cos (2 \pi \pi v-\varphi) \\
& =\mathrm{b}_{0}+\mathrm{b}_{1} \mathrm{~A}_{\mathrm{c}}(\mathrm{v}) \cos (2 \pi \pi v)+\mathrm{b}_{1} \mathrm{~A}_{\mathrm{s}}(v) \sin (2 \pi \pi v)
\end{aligned}
$$

Where

$$
A(v)=\left[A_{0}{ }^{2}(v)+A_{s}{ }^{2}(v)\right]^{1 / 2}
$$

And

$$
\begin{gathered}
\mathrm{A}_{c}(v)=\frac{\int \mathrm{A}(\delta(\cos (2 \pi 2 \pi v \delta)}{\int \mathrm{A}(\delta(\delta)} \\
\mathrm{A}_{s}(v) \frac{\int \mathrm{A}(\delta(\delta) \sin (v \delta) \mathrm{d} \delta}{\int \mathrm{A}(\delta(\delta)} \\
\cos \varphi=\frac{A_{\cdot}(v)}{A(v)} \\
\tan \varphi=\frac{A_{c}(v)}{A_{s}(v)}
\end{gathered}
$$

It is noted that the resulting image energy distribution $F(x)$ is still modulated by a cosine function of the same frequency $v$, demonstrating that a cosine distribution object is always imaged as a cosine distribution image. If the line spread function A $(\delta)$ is asymmetrical, a phase shift $\varphi$ is introduced.

The modulation in the image is given by

$$
M_{i}=\frac{b_{1}}{b_{0}}|A(v)|=M_{0}|A(v)|
$$

And $|A(v)|$ is the modulation transfer function

$$
\operatorname{MTF}(v)=|A(v)|=\frac{M_{i}}{M_{o}}
$$

The optical transfer function (OTF) is the complex function, which describes this process. It is a function of the spatial frequency $v$ of the sine-wave pattern. The real part of the OTF is the modulation transfer function (MTF) and the imaginary part is the phase transfer function (PTF). If the PTF is linear with frequency, it is, of course, just a simple lateral displacement of the 
image (as, for example, distortion), but if it is nonlinear, it can have an effect on the image quality. A phase shift of $180^{\circ}$ is a reversal of contrast, in that the image pattern is light where it should be dark, and vice versa. $[5,6]$

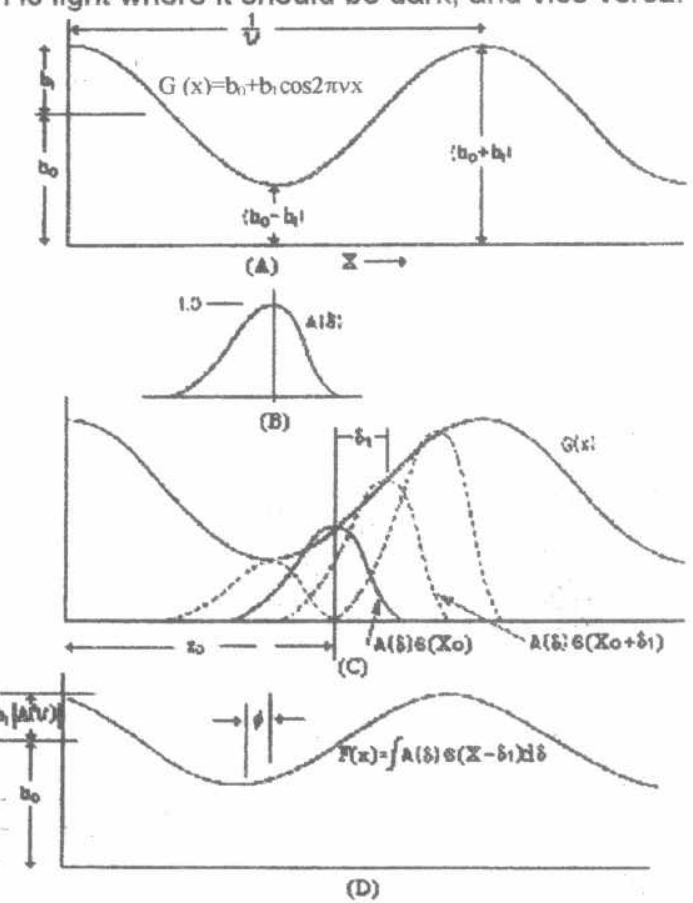

Fig. 2 Convolution of the object brightness distribution function $G(x)$ with the line spread function $A(\delta)$

\section{SOFTWARE PACKAGE FOR COMPUTING AND PLOTING of MTF}

In the following section a complete flowchart of the software is given which indicates the steps of tracing a bundle of rays through the typical IR seeker under test, a complete definition of the elements and dimensions of the typical IR seeker in fig (3), then the spot diagram and point spread function of the system are calculated and plotted. Finally, the MTF is calculated for each optical element.

By cascading the MTFs the total MTF is achieved. 


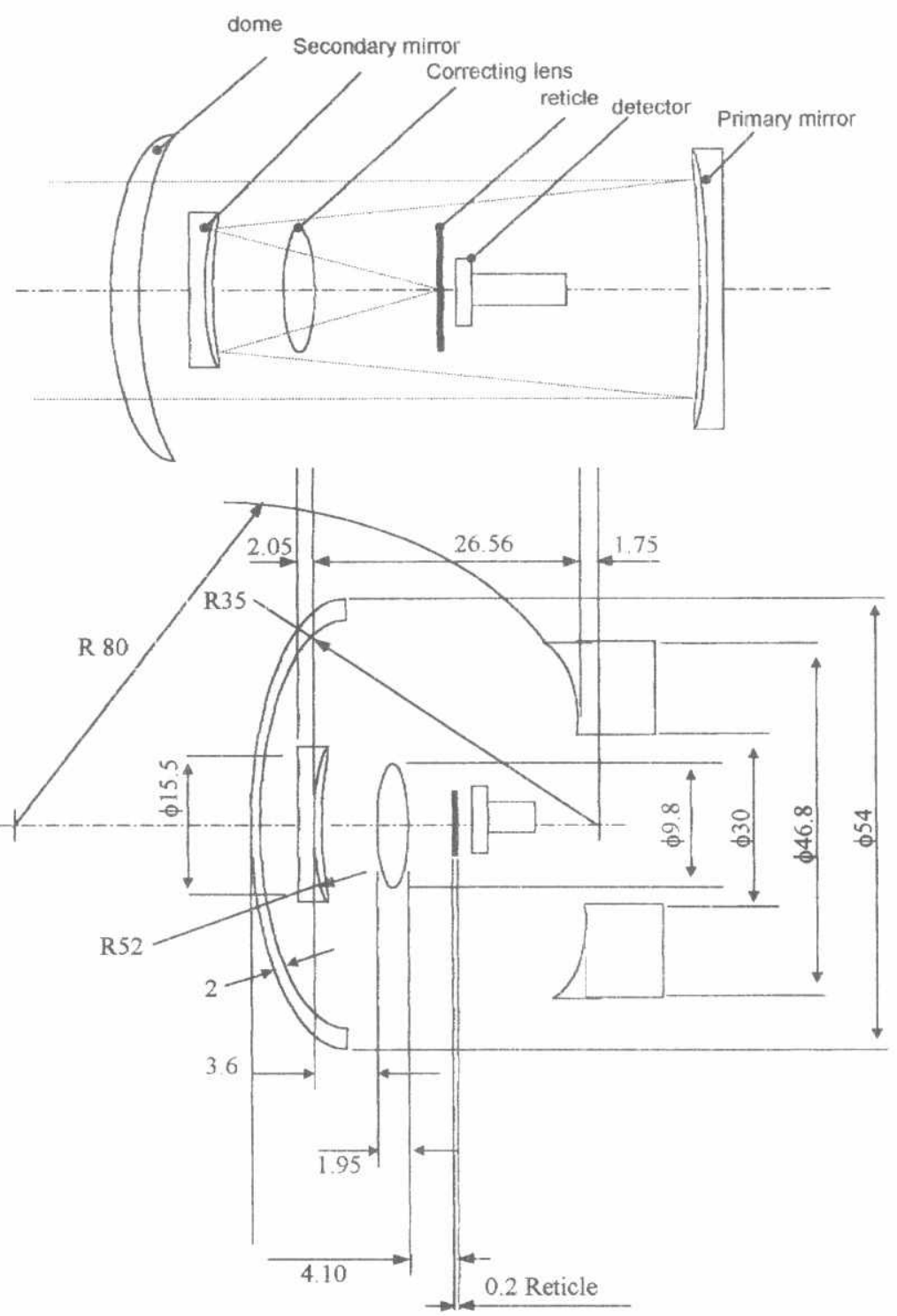

Fig 3 optical layout of the typical seeker 


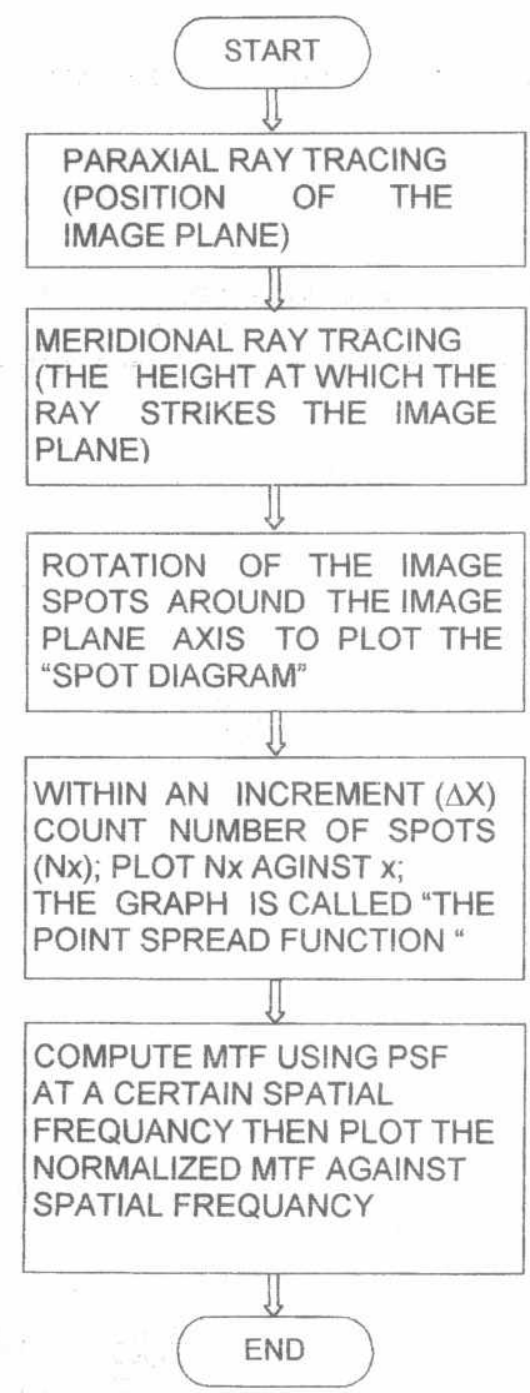

Fig 4 Flow Chart 


\section{OUTPUT OF THE SOFTWARE PACKAGE}

A sample of the output of the software package is given below. Each optical element will have three diagrams, which are very helpful in modeling its performance. A typical IR seeker is taken to be a case study in which we will model the performance of the optical element to test the performance of the homing head.

\section{The Dome}
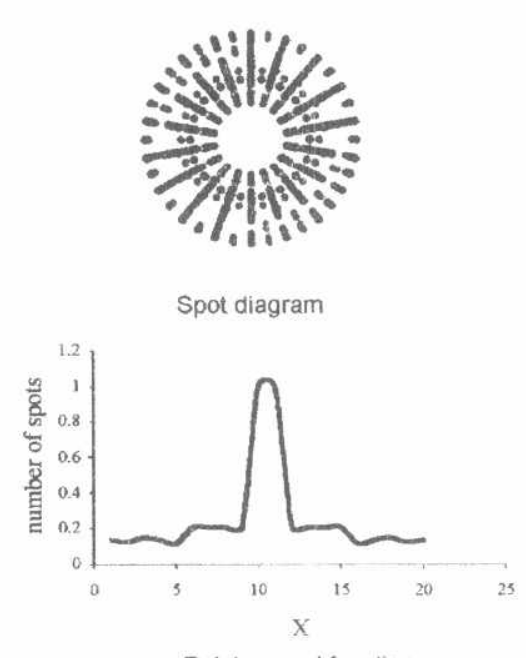

Point spread function

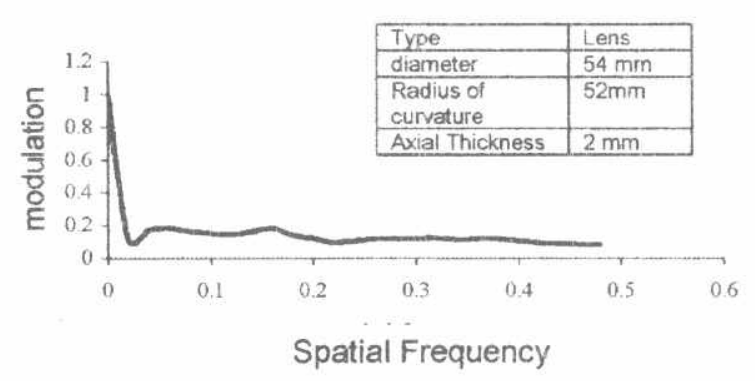

MTF of the dome 
The Primary Mirror
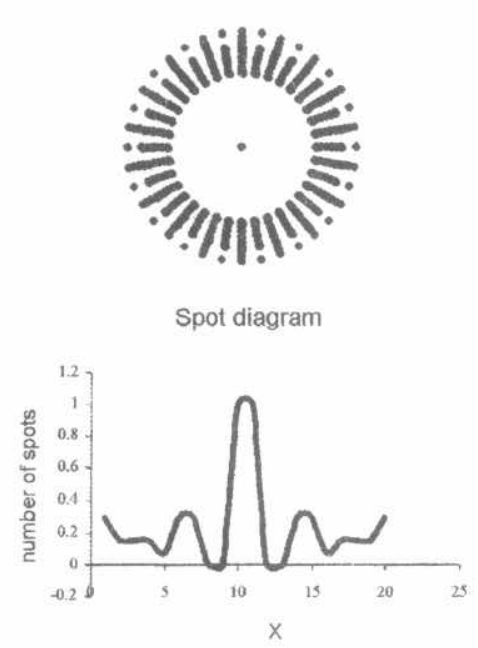

Point spread function

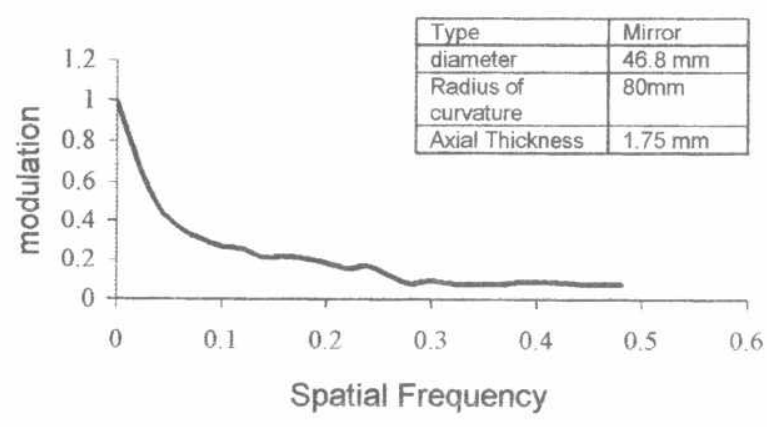

MTF of the primary mirror 
The secondary mirror

Spot diagram

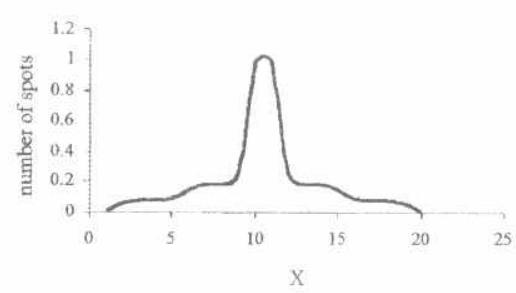

Point spread function

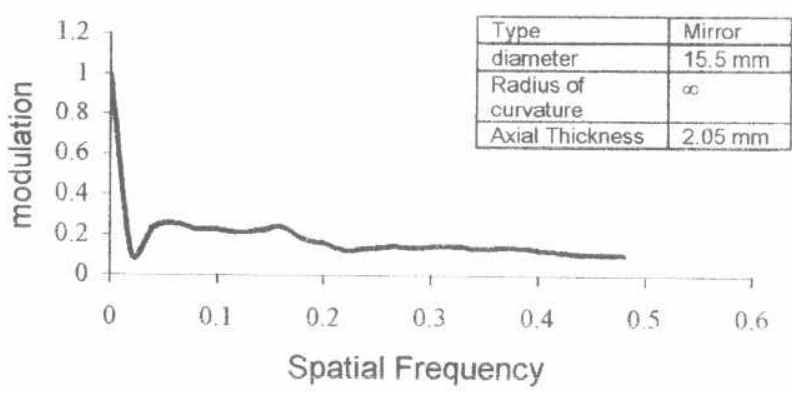

MTF of the secondary mirror 


\section{The Correcting Lens}

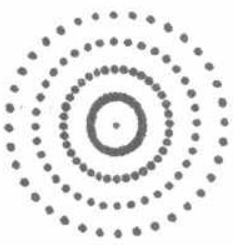

Spot diagram

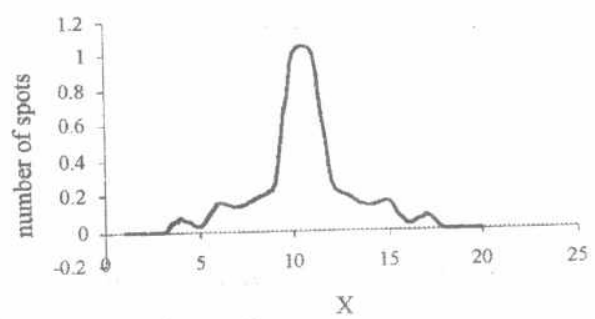

Point spread function

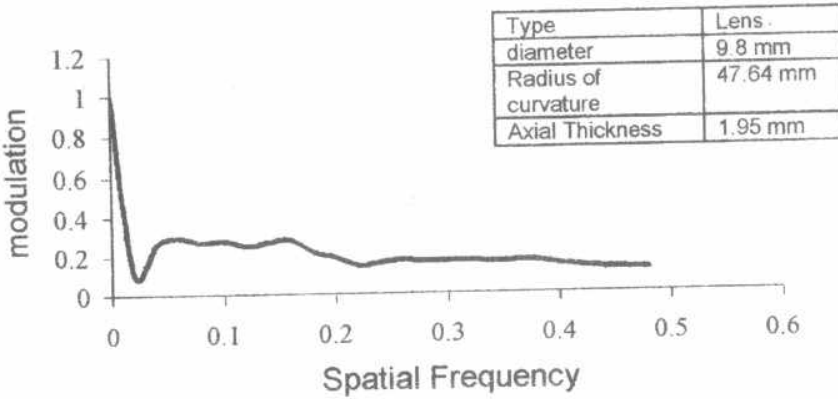




\section{CONCLUSION}

Using computers is a very powerful tool that minimizes efforts and costs spent in evaluation and modeling the optical systems. After finishing the modeling of each optical item i.e. MTF is achieved, the total MTF is calculated and plotted through cascading the MTFs of the items together. The results of the new software package will allow the designer to determine the best dimensions and shapes of the optical item, which will give excellen performance and hence minimum aberrations. Improvement of optical system performance and adding focal plane array detectors can be used in terminal guidance to enhance the resolution and hence the final missile-target missdistance.

\section{REFERENCE}

[1] Herzberger "Modern Geometrical Optics", New York, 1958.

[2] Smith, in W Drisoll (ed.)" Handbook Of Optics", New York, 1978.

[3] Smith, in Wolf and Zissis (eds) "The Infrared Handbook", Washington,

D.C., Office of Naval Research, 1985.

[4] M.Laikin "Lens Design", Marcel Dekker Inc., New York, 1991

[5] Levi and Austing, Applied Optics, Vol. 7, Optical society of America, Washington, D.C., 1968.

[6] C.S.Williams, O.A.Becklund," Optical Transfer Function", Joh Wiley, New York, 1989 